\title{
Correction to: The determinants of student loan take-up in England
}

\section{Ariane de Gayardon ${ }^{1} \cdot$ Claire Callender $^{2}$ - Francis Green ${ }^{1}$}

Published online: 21 June 2019

(C) Springer Nature B.V. 2019

\section{Correction to: Higher Education \\ https://doi.org/10.1007/s10734-019-00381-9}

The original version of this article unfortunately contained mistakes. Below are the corrections:

1. On page 9, "83\%" should be changed to "84\%" in this line "However, because most students take out both types of loans $(84 \%$ of borrowers in...."

2. There were typesetting errors in Tables 1,2 and 3. The corrected tables are now shown in the next pages.

The original article has been corrected.

The online version of the original article can be found at https://doi.org/10.1007/s10734-019-00381-9

Ariane de Gayardon

a.gayardon@ucl.ac.uk

1 Centre for Global Higher Education, UCL Institute of Education, 20 Bedford Way, London WC1H OAL, UK

2 UCL Institute of Education and Birkbeck, 26 Russell Square, London WC1B 5DQ, UK 
Table 1 Descriptive statistics of student loan borrowers by type of loan

\begin{tabular}{|c|c|c|c|c|}
\hline & $\begin{array}{l}\text { No student } \\
\text { loan }\end{array}$ & $\begin{array}{l}\text { Any student } \\
\text { loan }\end{array}$ & $\begin{array}{l}\text { Tuition fee } \\
\text { loan }\end{array}$ & $\begin{array}{l}\text { Maintenance } \\
\text { loan }\end{array}$ \\
\hline \multicolumn{5}{|l|}{ Family social class } \\
\hline $\begin{array}{l}\text { Higher managerial and professional } \\
\text { occupations }\end{array}$ & 0.12 & 0.88 & 0.82 & 0.80 \\
\hline $\begin{array}{l}\text { Lower managerial and professional } \\
\text { occupations }\end{array}$ & 0.09 & 0.91 & 0.84 & 0.85 \\
\hline Intermediate occupations & 0.07 & $0.93 *$ & 0.88 & 0.85 \\
\hline Small employers and own account workers & 0.12 & 0.88 & 0.82 & 0.81 \\
\hline Lower supervisory and technical occupations & 0.05 & $0.95 *$ & 0.90 & 0.83 \\
\hline Semi-routine occupations & 0.11 & 0.89 & 0.87 & 0.83 \\
\hline Routine occupations & 0.05 & $0.95 *$ & 0.92 & 0.85 \\
\hline Never worked/long-term unemployed & 0.11 & 0.89 & 0.85 & 0.77 \\
\hline \multicolumn{5}{|l|}{ Family's highest educational level } \\
\hline Degree or higher & 0.10 & 0.90 & 0.86 & 0.82 \\
\hline Less than a degree & 0.10 & 0.90 & 0.83 & 0.83 \\
\hline \multicolumn{5}{|l|}{ Family housing tenure analysis } \\
\hline Owned outright & 0.18 & 0.82 & 0.75 & 0.73 \\
\hline Being bought on a mortgage/bank loan & 0.08 & $0.92 *$ & 0.87 & 0.85 \\
\hline Other & 0.07 & $0.93 *$ & 0.90 & 0.83 \\
\hline \multicolumn{5}{|l|}{ School type } \\
\hline Public schooling & 0.08 & 0.92 & 0.87 & 0.84 \\
\hline Private schooling ${ }^{1}$ & 0.19 & $0.81^{*}$ & 0.74 & 0.75 \\
\hline \multicolumn{5}{|l|}{ Gender } \\
\hline Male & 0.08 & 0.92 & 0.87 & 0.84 \\
\hline Female & 0.11 & $0.89^{*}$ & 0.83 & 0.81 \\
\hline \multicolumn{5}{|l|}{ Ethnicity } \\
\hline White & 0.10 & 0.90 & 0.85 & 0.84 \\
\hline Mixed & 0.10 & 0.90 & 0.88 & 0.81 \\
\hline Indian & 0.15 & 0.85 & 0.81 & 0.71 \\
\hline Pakistani & 0.09 & 0.91 & 0.85 & 0.76 \\
\hline Bangladeshi & 0.06 & 0.94 & 0.90 & 0.69 \\
\hline Black Caribbean & 0.07 & 0.93 & 0.90 & 0.82 \\
\hline Black African & 0.05 & $0.95^{*}$ & 0.92 & 0.86 \\
\hline Other & 0.09 & 0.91 & 0.89 & 0.78 \\
\hline \multicolumn{5}{|l|}{ Religion } \\
\hline No religion & 0.08 & 0.92 & 0.87 & 0.85 \\
\hline Muslim & 0.11 & 0.89 & 0.85 & 0.70 \\
\hline Other & 0.10 & 0.90 & 0.84 & 0.83 \\
\hline \multicolumn{5}{|l|}{ Living at home } \\
\hline Never & 0.07 & 0.93 & 0.87 & 0.88 \\
\hline Partially & 0.07 & 0.93 & 0.87 & 0.87 \\
\hline Always & 0.17 & $0.83^{*}$ & 0.78 & 0.66 \\
\hline \multicolumn{5}{|l|}{ Working during term-time } \\
\hline No work & 0.11 & 0.89 & 0.83 & 0.82 \\
\hline Irregular & 0.08 & 0.92 & 0.85 & 0.84 \\
\hline Regular & 0.09 & 0.91 & 0.86 & 0.82 \\
\hline \multicolumn{5}{|l|}{$\mathrm{IDACI}^{2}$} \\
\hline Mean score & $0.14(0.15)$ & $0.16(0.18)^{*}$ & $0.16(0.18)$ & $0.16(0.17)$ \\
\hline \multicolumn{5}{|l|}{ Permanent equivalised income (in $£ 10,000)^{2}$} \\
\hline Mean score & $2.50(1.90)$ & $2.01(1.54)^{*}$ & $1.96(1.50)$ & $2.00(1.49)$ \\
\hline \multicolumn{5}{|l|}{ Debt attitude index ${ }^{2}$} \\
\hline Mean score & $12.97(2.89)$ & $13.68(2.96)^{*}$ & $13.70(3.00)$ & $13.74(2.93)$ \\
\hline Observations $(\mathrm{N})$ & 356 & 3932 & 3500 & 3318 \\
\hline
\end{tabular}

These estimates use the survey-supplied weights for wave 7. They are calculated for the sample of students who attended higher education by wave 7 . Estimates are provided for the students with debt or without, as well as separately for those who took out maintenance loans and for those who took out tuition fee loans

* indicates a significant difference between debtors and non-debtors for continuous variables; for categorical variables, * indicates a significant difference between the marked category and the first category of the variable

${ }^{1}$ Private schooling refers to fees paying secondary schools

${ }^{2}$ For continuous variables, we give the mean and standard deviation in parenthesis 
Table 2 Probit model of student loan take-up
(1)
(2)
(3)

Family social class $($ base $=$ all else)

Higher managerial and professional

$-0.0235(-0.21) \quad-0.0176(-0.16) \quad-0.0511(-0.44)$ occupations

Family's highest educational level (base $=$ below first degree)

Higher education first degree or more

$0.224 * *(2.69)$

Family housing tenure (base $=$ own outright)

Being bought on mortgage/bank loan

Other

Type of school at 13 (base $=$ maintained $)$

Private schooling

IDACI (standardised)

Permanent equivalised income (in $£ 10,000$ )

Gender $($ base $=$ male $)$

Female

Ethnicity (base $=$ White)

Mixed

Indian

Pakistani

Bangladeshi

Black Caribbean

Black African

Other

Religion (base $=$ none $)$

Muslim

Other

Debt attitude index

Living at home while in HE (base = never)

Partially lived at home while in HE

Always lived at home while in $\mathrm{HE}$

$\begin{array}{lll}0.489 * * *(5.93) & 0.467 * * *(5.69) & 0.482 * * *(5.69) \\ 0.487 * *(3.11) & 0.480 * *(3.05) & 0.510 * *(3.26)\end{array}$

$-0.321 * * * \quad-0.339 * * * \quad-0.434 * * *$

$$
\begin{array}{lll}
(-3.48) & (-3.69) & (-4.78)
\end{array}
$$

$0.0873(1.47) \quad 0.0886(1.50) \quad 0.157 * *(2.64)$

$-0.0959 * * \quad-0.102 * *(-2.92)-0.101 * *(-2.87)$

$(-2.76)$

$-0.211 * *(-3.24)-0.183 * *(-2.84)-0.199 * *(-3.01)$

$-0.0906(-0.46) \quad-0.0745(-0.37) \quad-0.0818(-0.43)$

$-0.309 *(-2.05) \quad-0.331 *(-2.20) \quad-0.184(-1.25)$

$\begin{array}{lll}0.455(1.57) & 0.420(1.45) & 0.556(1.81)\end{array}$

$0.488 *(2.11) \quad 0.473 *(2.04) \quad 0.594 *(2.57)$

$-0.147(-0.65) \quad-0.123(-0.54) \quad 0.0175(0.07)$

$\begin{array}{lll}-0.0555(-0.27) & -0.0535(-0.25) & -0.132(-0.61)\end{array}$

$\begin{array}{lll}0.277(0.96) & 0.193(0.83) & 0.266(1.14)\end{array}$

$-0.605 * *(-2.94)-0.591 * *(-2.85)-0.401(-1.93)$

$\begin{array}{lll}-0.0734(-0.91) & -0.0649(-0.82) & -0.0450(-0.56)\end{array}$

$0.0552 * * *(3.96) \quad 0.0465 * *(3.16)$

Working during term-time (base $=$ no work)

Irregular work

$-0.0983(-0.67)$

$-0.698 * * *$

$(-7.62)$

Regular work

Constant

Observations, 3754

$0.0907(0.91)$

$0.125(1.47)$

$1.451 * * *(11.98) \quad 0.728^{* *}(3.26) \quad 1.024 * * *(4.22)$

Reported in this table are the raw coefficients for the model and $\mathrm{t}-$ statistics in parenthesis. These estimates use the survey-supplied weights for wave 7 . The outcome is a dummy variable that takes the value 1 if the individual student has taken out any type of student loans

$* p<0.05 ; * * p<0.01 ; * * * p<0.001$ 


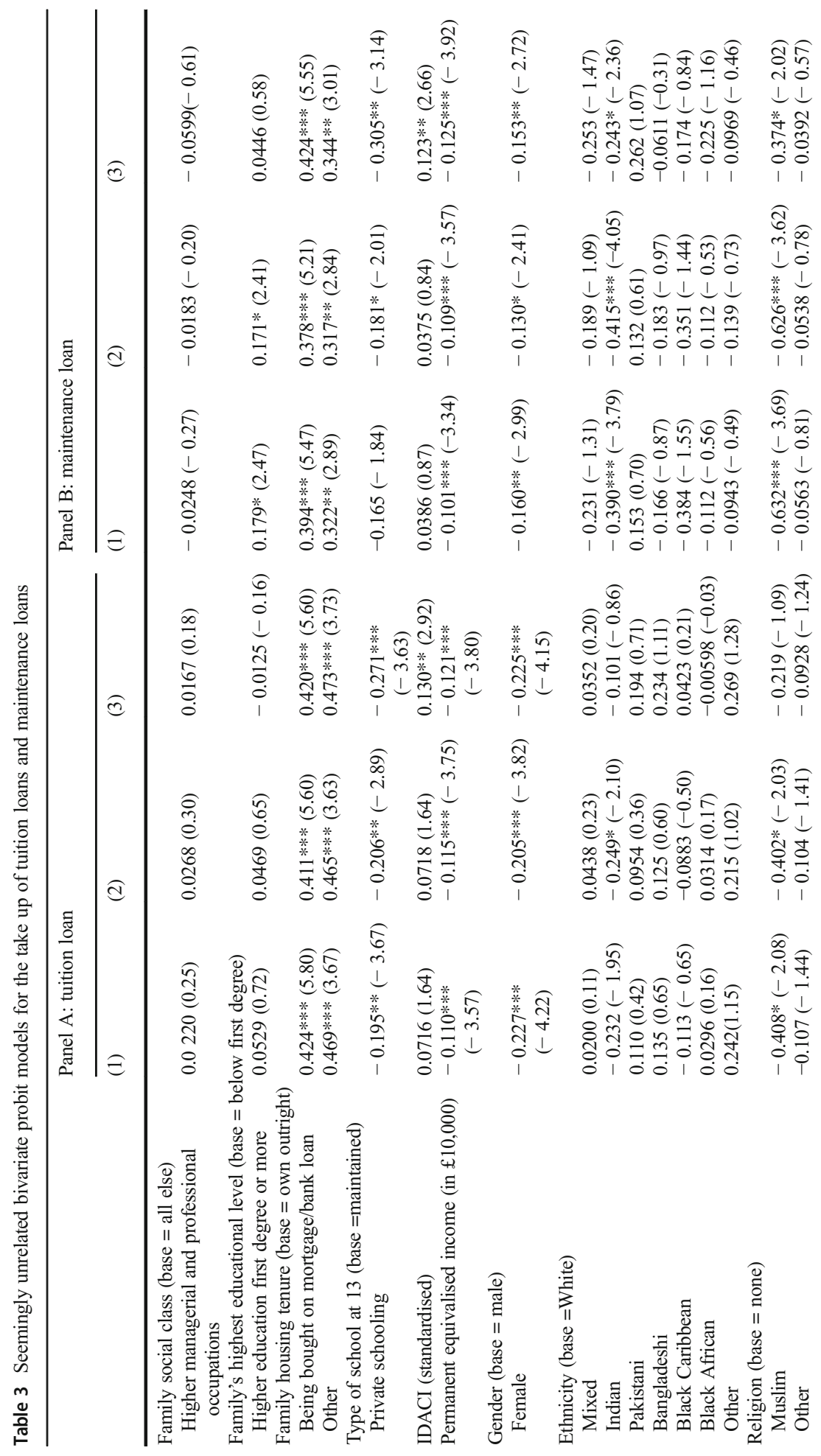




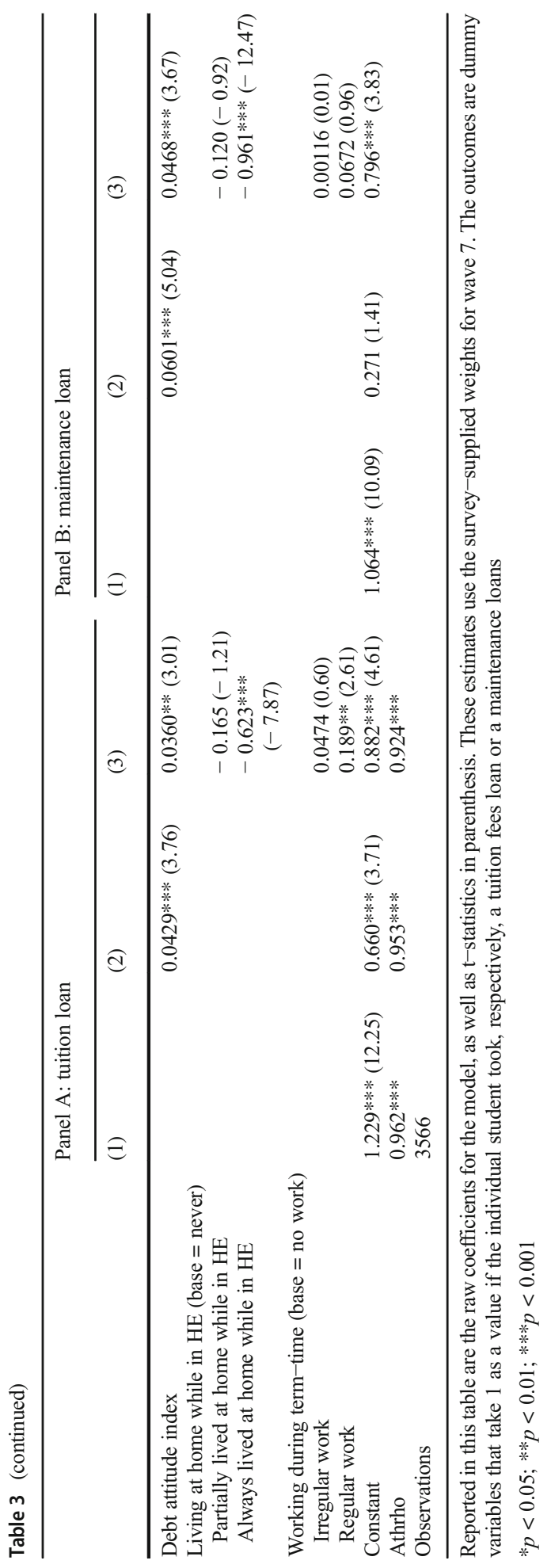


Publisher's note Springer Nature remains neutral with regard to jurisdictional claims in published maps and institutional affiliations. 